hep-th/9805200

CERN-TH:98-95

KCL-MTH:98-12

LPTHE:98-16

\title{
Six-Dimensional TQFTs and Twisted Supersymmetry
}

\author{
Laurent Baulieu \\ CERN, Geneva, Switzerland \\ and \\ LPTHE目, Paris, France.
}

Peter West

King's College, Strand, London WC2 RLS, England.

\begin{abstract}
We describe a generalization of Yang-Mills topological field theory for Abelian two-forms in six dimensions. The connection of this theory by a twist to Poincaré supersymmetric theories is given. We also briefly consider interactions and the case of self-dual three-forms in eight dimensions.
\end{abstract}

1 UMR CNRS associée aux Universités Pierre et Marie Curie (Paris VI) et Denis Diderot (Paris VII). 


\section{Introduction}

In this paper we introduce a generalization of topological field theory in six dimensions for two-form gauge fields. We analyse this theory using the techniques of BRST quantization for Topological Quantum Field Theories (TQFT) thus generalizing to six dimensions the Yang-Mills TQFTs in four dimensions studied in [1] [2] [3] [4]. In section 2 we determine the topological BRST operator $Q$ and show that the result is related to a Poincaré supersymmetric theory in six dimensions with $(2,0)$ supersymmetry. We explain how $Q$ must be combined with the BRST operator of the ordinary degenerate gauge symmetry of twoforms to obtain a completely gauge-fixed action for the TQFT. We also briefly comment on the Hamiltonian presentation of the TQFT, in the spirit of [1]. In section 3, we show the possibility of introducing interactions, either by considering couplings of real two-forms to a Yang-Mills field by a Chern-Simons term. We finish by noting that our results can be generalized to eight dimensions where the two-form is replaced by a three-form and the corresponding twist leads to a supersymmetric theory that contains a gravitino, but no graviton.

\section{Free Case}

\subsection{The fields and the $Q$-symmetry.}

Our aim is to construct a TQFT in six dimensions for two-form gauge fields $B_{2}=$ $\frac{1}{2} B_{\mu \nu} d x^{\mu} \wedge d x^{\nu}$. We chose the Minkowskian signature for the six-dimensional space. (Our formulae can be adapted to the Euclidian case, or other signatures, with minor modification in the equations.) Our basic hypothesis is that the TQFT explores quantum fluctuations around the solution of the self-duality equations between two independant real two-forms gauge fields $B_{2}$ and ${ }^{c} B_{2}$

$$
\partial_{[\mu} B_{\nu \rho]}+\epsilon_{\mu \nu \rho \alpha \beta \gamma} \partial_{[\alpha}{ }^{c} B_{\beta \gamma]}=0 .
$$

Having a pair of two-forms is necessary in order to define a "topological" invariant $I_{c l}$ that does not vanish trivially (the exterior product of a three-form by itself is zero). We take for $I_{c l}$ :

$$
I_{c l}=\int d B_{2} \wedge d^{c} B_{2}
$$

This invariant can be non trivial. Indeed, $B_{2}$ and ${ }^{c} B_{2}$ can be defined as "connections" rather than as forms, and such that $d B_{2}$ and $d^{c} B_{2}$ are still forms and thus are integrable. 
(See [5]). Then, the integral (2.2) is well-defined, but can be non zero. Actually, up to a normalization, (2.2) must be $\mathcal{Z}$-valued. This may appear as a motivation for the construction for a TQFT for two-forms in six dimensions.

We notice that the gauge condition (2.1) implies self-duality and antiself-duality relations for the curvatures of $B_{2}+{ }^{c} B_{2}$ and $B_{2}-{ }^{c} B_{2}$ respectively. As such, it imposes for ten independent conditions separately for each gauge field combination. We will use the left hand side of (2.1) as gauge functions for the BRST-invariant gauge-fixing of $I_{c l}$, since the number of gauge-independent components of the two-forms $B_{2}$ and ${ }^{c} B_{2}$ in six dimensions is precisely $2 C_{5}^{2}=20$.

The theory must exhibit a topological BRST symmetry with a generator $Q$. $Q$ is a graded differential operator acting on the gauge-invariant sector of the TQFT, whose fields are defined in the following expansion (the upper index is the ghost number)

$$
\begin{aligned}
& F_{\mu \nu \rho} d x^{\mu} \wedge d x^{\nu} \wedge d x^{\rho}+\Psi_{\mu \nu}^{1} d x^{\mu} \wedge d x^{\nu}+\Psi_{\mu \nu}^{-1} d x^{\mu} \wedge d x^{\nu} \\
& +\Phi_{\mu}^{2} d x^{\mu}+\Phi_{\mu}^{-2} d x^{\mu}+L_{\mu} d x^{\mu}+\Phi^{3}+X^{1}+X^{-1}+\Phi^{-3}
\end{aligned}
$$

The other two-form ${ }^{c} B_{2}$ possess an identical expansion (2.3) and the foregoing equations must be duplicated in an obvious way.

A geometrical signification presumably exists (as suggested by the formula (2.29-2.30) of section 2.4) for the ghosts $\Psi_{\mu \nu}^{1}, \Phi_{\mu}^{2}, \Phi^{3}$. For these fields, one has:

$$
\begin{array}{ll}
Q B_{\mu \nu}=\Psi_{\mu \nu}^{1} & Q \Psi_{\mu \nu}^{1}=\partial_{[\mu} \Phi_{\nu]}^{2} \\
Q \Phi_{\mu}^{2}=\partial_{\mu} \Phi^{3} & Q \Phi^{3}=0
\end{array}
$$

$Q$ is nilpotent up to a (degenerate) gauge transformation of $B_{2}$, with "parameter" $\Phi_{\mu}^{2}$. It can be used to define the equivariant cohomology of two-forms, modulo their gauge transformations which are discussed in section 2.4.

The rest of the fields in (2.3) (not including the classical curvature $F_{\mu \nu \rho}$ of the two-form $B_{\mu \nu}$ ) are antighosts, which are related to antifields in the Batalin-Vilkoviski formalism (see for instance [6]) and transform into auxiliary (Lagrange multiplier) fields:

$$
\begin{array}{lrrr}
Q \Psi_{\mu \nu}^{-1}=H_{\mu \nu} & Q H_{\mu \nu}=0 & Q \Phi_{\mu}^{-2}=\eta_{\mu}^{-1} & Q \eta_{\mu}^{-1}=0 \\
Q L_{\mu}=\eta_{\mu}^{1} & Q \eta_{\mu}^{1}=0 & Q \Phi^{-3}=\eta^{-2} & Q \eta^{-2}=0 \\
Q X^{-1}=\eta & Q \eta=0 & Q X^{1}=\eta^{2} & Q \eta^{2}=0 .
\end{array}
$$

Our purpose is to define a theory whose topological gauge conditions are the $20=10+10$ self-duality conditions for $B_{2}$ and ${ }^{c} B_{2}$. To do so, we need to introduce 10 self-dual and 10 
antiself-dual three-form Lagrange multipliers. These must be defined from the $15+15$ fields $H_{\mu \nu}$ and ${ }^{c} H_{\mu \nu}$ Following the spirit of [3], this leads us to decompose the two-form antighosts $\Psi_{\mu \nu}^{-1}$ with fifteen components into a self-dual three-form $\chi_{\mu \nu \rho}^{-1}$ with ten components and a field $\chi_{L}^{-1}$ with five components $\chi_{L(a)}^{-1}$, where $a$ runs from 1 to 5 . (This decomposition is not Lorentz covariant at each step, but the result is the Lorentz covariant BRST multiplet (2.9).) The same is done for the Lagrange multipliers that are the BRST transforms of the antighosts, which gives $H_{\mu \nu \rho}$ and $H_{L}$, with $H_{\mu \nu \rho}=\left\{Q, \chi_{\mu \nu \rho}^{-1}\right\}$ and $H_{L}=\left\{Q, \chi_{L}^{-1}\right\}$. For the second set of fields with upper index ${ }^{c}$, we take ${ }^{c} \chi_{\mu \nu \rho}^{-1}$ and ${ }^{c} H_{\mu \nu \rho}$ as antiself-dual three-forms.

The antighosts and Lagrange multipliers $X^{-1}, \chi_{L(a)}^{-1}, \eta_{\mu}^{1}, \eta, H_{L(a)}$ and $L_{\mu}$ must be eliminated from the TQFT. This can be done by a brute-force gauge-fixing, obtained from the following $Q$-exact Lagrangian:

$$
\left\{Q, X^{-1} L_{6}+\sum_{a=1}^{5} \chi_{L(a)}^{-1} L_{a}\right\}=\eta L_{6}-X^{-1} \eta_{6}^{1}+\sum_{a=1}^{5}\left(H_{L(a)} L_{a}-\chi_{L(a)}^{-1} \eta_{a}^{1}\right) .
$$

The equations of motion are delta functions that provide the desired gauge-fixing to zero of the fields $X^{-1}, \chi_{L(a)}^{-1}, \eta_{\mu}^{1}, \eta, H_{L(a)}$ and $L_{\mu}$. Their elimination can be rephrased as follows: the -5 degrees of freedom of the fermions $\chi_{L(a)}^{-1}$ are compensated by the +6 degrees of freedom of the bosons $L_{\mu}$ plus the -1 degree of freedom of the fermion $X^{-1}$; said differently, a self-dual three-form $\chi_{\mu \nu \rho}^{-1}$ in six dimensions is the combination of the fields $\left(\Psi_{\mu \nu}^{-1}, L_{\mu}, X^{-1}\right)$ with algebraic compensations between commuting and anticommuting fields.

We thus reduce the expansion (2.3) and the definition (2.5) of $Q$ as follows

$$
\begin{aligned}
& F_{\mu \nu \rho} d x^{\mu} \wedge d x^{\nu} \wedge d x^{\rho}+\Psi_{\mu \nu}^{1} d x^{\mu} \wedge d x^{\nu}+\chi_{\mu \nu \rho}^{-1} d x^{\mu} \wedge d x^{\nu} \wedge d x^{\rho} \\
& +\Phi_{\mu}^{2} d x^{\mu}+\Phi_{\mu}^{-2} d x^{\mu}+\Phi^{3}+X^{1}+\Phi^{-3} \\
& Q B_{\mu \nu}=\Psi_{\mu \nu}^{1} \quad Q \Psi_{\mu \nu}^{1}=\partial_{[\mu} \Phi_{\nu]}^{2} \quad Q \Phi_{\mu}^{2}=\partial_{\mu} \Phi^{3} \quad Q \Phi^{3}=0 \\
& Q \chi_{\mu \nu \rho}^{-1}=H_{\mu \nu \rho} \quad Q H_{\mu \nu \rho}=0 \quad Q \Phi_{\mu}^{-2}=\eta_{\mu}^{-1} \quad Q \eta_{\mu}^{-1}=0 \\
& Q X^{1}=\eta^{2} \quad Q \eta^{2}=0 \quad Q \Phi^{-3}=\eta^{-2} \quad Q \eta^{-2}=0 .
\end{aligned}
$$

The bosonic fields $H_{\mu \nu \rho}$ and $\eta^{ \pm 2}$ will eventually be eliminated as Lagrange multipliers for gauge conditions. Therefore, the "basic" TQFT multiplet for a two-form with selfduality gauge conditions is

$$
\left(B_{\mu \nu}, \Psi_{\mu \nu}^{1}, \chi_{\mu \nu \rho}^{-1}, \eta_{\mu}^{-1}, \Phi^{3}, \Phi^{-3}, X^{1}, \Phi_{\mu}^{2}, \Phi_{\mu}^{-2}\right)
$$


This multiplet could have been our starting point, in particular in the Hamiltonian construction sketched in section 3. Since we have in addition the fields arising from the gauge field ${ }^{c} B_{\mu \nu}$ we have in effect twice the number of the above fields in the actual topological theory. Let us again stress that in (2.9), $B_{\mu \nu}$ is unconstrained. The TQFT multiplet of ${ }^{c} B_{\mu \nu}$ is completely analogous to (2.9), except that ${ }^{c} \chi_{\mu \nu \rho}^{-1}$ is antiself-dual rather than self-dual.

\subsection{Twist.}

The multiplet (2.9) contains $34=15+10+6+3$ fermionic degrees of freedom and $15+12$ bosonic degrees of freedom. To make the link to Poincaré supersymmetry, we assume that two bosonic and two fermionic degrees of freedom, e.g $\Phi^{ \pm 3}$ and $\Phi_{6}^{ \pm 2}$, compensate each other by supersymmetry, as two topological packages in the language of [7]. What remains is a system of 32 fermions and $15+10$ bosons, which we write as

$$
\left(B_{\mu \nu}, \Psi_{\mu \nu}^{1}, \chi_{\mu \nu \rho}^{-1}, \eta_{\mu}^{-1}, X^{1}, \Phi^{a}\right)
$$

with $1 \leq a \leq 10$.

We now wish to find a supersymmetric multiplet that, after a twist, can be identified with the above fields. Since the fields in the above equation do not include a graviton this supersymmetric multiplet must possess supersymmetry transformations with less than 32 supercharges. As we are in six dimensions the largest possible number of supercharges that is less than 32 is 16 . The supermultiplet with this number of supercharges and a second-rank antisymmetric tensor gauge field is $(2,0)$ tensor multiplet [8].

Before giving the details of the twist in six dimensions it will be instructive to recall some of the essential features of the twist to relate the topological Yang-Mills theory in four dimensions to the supersymmetry multiplet of $N=2$ supersymmetric Yang-Mills theory [2]. The $N=2, D=4$ supersymmetric multiplet is given by $\left(A_{m}, \lambda_{\alpha}^{i}, \bar{\lambda}_{\dot{\alpha} i}, B, \bar{B}\right)$ and it is related by the twist to the TQFT multiplet $\left(A_{m}, \Psi_{m}^{(1)}, \chi_{m n}^{(-1)}, \eta^{(-1)}, \Phi^{(2)}, \Phi^{(-2)}\right)$ in the notation of ref. [2]. The essential step in the twist proceedure is the identification of the $S U(2)_{\text {susy }}$ and $S U(2)_{R}$ groups in the $S O(4) \times S U_{\text {susy }}(2) \sim S U_{L}(2) \times S U(2)_{R} \times S U(2)_{\text {susy }}$ invariance of the SSYM theory [1] [2].

After setting the supersymmetry parameter to $\eta^{\alpha i}=0, \bar{\eta}^{\dot{\alpha} \dot{\beta}}=-\epsilon^{\dot{\alpha} \dot{\beta}} \rho$ we find [2] that the $N=2$ Yang-Mills transformations for $A_{\mu}, \lambda_{\alpha \dot{\beta}}=\left(\sigma^{m}\right)_{\alpha \dot{\beta}} \Psi_{m}^{(1)}$ and $\Phi^{(2)} \equiv B$ give the correct transformations for the original gauge fields and the ghosts. However, this 
discussion can be slightly extended to recover also the BRST transformations of the antighosts and Lagrange multipliers. Indeed, making the identifications $\bar{\lambda}_{\dot{\alpha} \dot{\beta}} \equiv\left(\sigma^{m n}\right)_{\dot{\alpha} \dot{\beta}} \chi_{m n}^{(-1)}+$ $\epsilon_{\dot{\alpha} \dot{\beta}} \eta^{(-1)}$ and $\bar{B} \equiv \Phi^{(-2)}$, we find that the supersymmetry transformations of eq. (3.4) of ref. [2] imply, up to constants, that $\delta \chi_{m n}^{(-1)}=\rho\left(F_{m n}-\frac{1}{2} \epsilon_{m n p q} F^{p q}\right), \delta \Phi^{(-2)}=\rho \eta^{(-1)}$ and $\delta \eta^{(-1)}=0$. These variations are the BRST transformations of the antighosts after the self-dual Lagrange multiplier has been eliminated to enforce the gauge condition and the auxiliary field in the $N=2$ Yang-Mills theory has been set to vanish.

Let us now consider how the fields and the transformation laws of the BRST symmetry (2.8) are related to those of the $(2,0)$ tensor multiplet of Poincaré supersymmetry in six dimensions. The six-dimensional spinors we are interested in transform under $\operatorname{Spin}(1,5) \times \operatorname{Spin}(5)$. The first group in this direct product is by definition the group under which a six-dimensional spinor transforms. The second group is an internal group that arises naturally for six-dimensional spinors that have their origin in eleven dimensions, where they transform under $\operatorname{Spin}(1,10)$. In fact, $\operatorname{Spin}(5)$ is isomorphic to $U S P(4)$. Spinors in six dimensions have $2^{3}=8$ components. The matrix $B$, which implements the relation $B \gamma^{\mu} B^{-1}=\gamma^{\mu^{*}}$ satisfies, $B^{\dagger} B=1$ and $\tilde{B}=-B$. As in any even-dimensional space we can have Weyl spinors, but the properties of $B$ imply that Majorana spinors (i.e. $\left.\psi^{*}=B \psi\right)$ do not exist in six dimensions. We work here not with the full $8 \times 8$ six-dimensional $\gamma$-matrices, but with their chirally projected components which are $4 \times 4$ matrices.

However, in six dimensions we can have symplectic Majorana-Weyl spinors, which have the indices $\kappa_{\alpha i}$ where the indices $\alpha=1, \ldots, 4$ transform under Weyl-projected $\operatorname{Spin}(1,5)$ transformations and the indices $i=1, \ldots, 4$ transform under $\operatorname{Spin}(5)$ transformations. The Majorana-Weyl condition reads

$$
\kappa_{\alpha i}^{*}=B_{\dot{\alpha}}^{\beta} \Omega^{i j} \kappa_{\beta j},
$$

where $\Omega^{i j}$ is the antisymmetric real metric of $U S P(4)$, which raises and lowers indices by $S^{i} \equiv \Omega^{i j} S_{j}$ and $S_{j}=S^{k} \Omega_{k j}$. The matrix $B$ is block diagonal. If we denote its upper- and lower-blocks by $B_{\dot{\alpha}}{ }^{\beta}$ and $B_{\dot{\gamma}}{ }^{\beta}$ respectively, we can convert dotted indices to undotted ones (i.e. $\chi_{\dot{\beta}}=B_{\dot{\beta}}^{\gamma} \chi_{\gamma}$ and $\chi^{\beta}=\chi^{\dot{\gamma}} B_{\dot{\gamma}}{ }^{\beta}$ ). We note, however, that we cannot raise and lower the spinor indices using the $B$ matrix.

The simplest supermultiplet that contains such a Majorana-Weyl spinor is the socalled $(2,0)$ tensor multiplet which possess the real fields $\Phi_{i j}, B_{\mu \nu}$ and spinors $\kappa_{\alpha i}$. The 
"on-shell" counting of degrees of freedom goes as follows: the $\kappa_{\alpha i}$ obey eq. (2.11) and so contribute 8 on-shell degrees of freedom; the five scalars live in the real field $\Phi_{i j}$, which obeys the constraints

$$
\Phi_{i j}=-\Phi_{j i}, \quad \Omega^{i j} \Phi_{i j}=0 .
$$

The gauge field $B_{\mu \nu}$ possesses a self-dual field strength $F_{\rho \mu \nu}^{(+)}=3 \partial_{[\rho} B_{\mu \nu]}$ (i.e. $F_{\rho \mu \nu}^{(+)}=$ $\left.\frac{1}{3 !} \varepsilon_{\rho \mu \nu \kappa \tau \kappa} F^{\kappa \tau \kappa(+)}\right)$, which provides only 3 on-shell degrees of freedom. This is the multiplet that arises in the 5-brane of M-theory. This $(2,0)$ supermultiplet transforms under supersymmetry with a parameter that is also Majorana-Weyl and so has the desired 16 components.

The supersymmetry variations of the component fields of the $(2,0)$ tensor multiplet are given, up to constants, by

$$
\begin{aligned}
& \delta \kappa_{\alpha i}=F_{\mu \nu \rho}^{(+)}\left(\gamma^{\mu \nu \rho}\right)_{\alpha \beta} \epsilon_{i}^{\beta}+\left(\gamma^{\mu} \partial_{\mu}\right)_{\alpha \dot{\beta}} \Phi_{i j} \bar{\epsilon}^{\dot{\beta} j} \\
& \delta \bar{\kappa}_{\dot{\alpha}}^{i}=\bar{F}_{\mu \nu \rho}^{(+)}\left(\gamma^{\mu \nu \rho}\right)_{\dot{\alpha} \dot{\beta}} \bar{\epsilon}^{\dot{\beta} i}+\left(\gamma^{\mu} \partial_{\mu}\right)_{\dot{\alpha} \beta} \bar{\Phi}^{i j} \epsilon^{\beta j} \\
& \delta B_{\mu \nu}=\epsilon_{i}^{\alpha}\left(\gamma_{\mu \nu}\right)_{\alpha}^{\dot{\beta}} \bar{\kappa}_{\dot{\beta}}^{i} \\
& \delta \Phi_{i j}=\epsilon_{[i}^{\beta} \kappa_{\beta j]}-\frac{1}{4} \Omega_{i j} \epsilon_{k}^{\beta} \kappa_{\beta}^{k} \\
& \delta \bar{\Phi}^{i j}=\bar{\kappa}_{\dot{\alpha}}^{[i} \bar{\epsilon}^{\alpha j]}+\frac{1}{4} \Omega^{i j} \bar{\kappa}_{\dot{\alpha}}^{k} \bar{\epsilon}_{k}^{\dot{\alpha}} .
\end{aligned}
$$

Examining the variation of the spinors, we find that the self-duality condition is associated with the Weyl nature of the spinors as the Weyl projected $\gamma$-matrix $\left(\gamma^{a b c}\right)_{\alpha \beta}$ is automatically antiself-dual. On the other hand, the reality condition of the scalars and the gauge field in the $(2,0)$ supermultiplet follows from the symplectic Majorana condition of eq. (2.11) when imposed on the supersymmetry variation of the spinors. Of course $\kappa$ and $\bar{\kappa}$ are related by their Majorana-Weyl condition, while $\Phi_{i j}$ and $\bar{\Phi}^{i j}$ are related by lowering their indices, but it will be convenient to write the transformation laws in this way.

We now wish to twist the above supersymmetry transformations and recover the topological BRST transformations. Clearly the six-dimensional spin group $\operatorname{Spin}(1,5)$ contains $\operatorname{Spin}(5)$. The twist consists in identifying these $\operatorname{Spin}(5)$ transformations with those of the internal Spin(5) symmetry group. The group $U S P(4)$ consists of unitary matrices that also preserve $\Omega^{i j}$, i.e. matrices $A$ that satisfy $A^{T} A=1$ and $A^{T} \Omega A=\Omega$. The latter condition can also be written as $\Omega A=A^{*} \Omega$. On the other hand the $\operatorname{Spin}(1,5)$ acts separately on each chiral sector and the chirally projected $\operatorname{Spin}(1,5)$ transformations are isomorphic to $S U^{*}(4)$, which is the group of four by four complex matrices of determinant 1 , which 
obey $B A=A^{*} B$. Hence to identify the two $\operatorname{Spin}(5)=U S P(4)$ groups also requires us to identify $\Omega=B$.

Having identified the two $\operatorname{Spin}(5)$ groups, we may place $\operatorname{Spin}(5)$ invariant conditions on the supersymmetry parameters $\varepsilon_{i}^{\alpha}$ and $\bar{\varepsilon}^{\dot{\alpha} i}$. In particular, we choose

$$
\bar{\varepsilon}^{\dot{\alpha} i}=0, \quad \varepsilon_{i}^{\alpha}=\delta_{i}^{\alpha} \rho
$$

We may write the latter constraint as $\varepsilon^{\alpha i}=\rho \Omega^{i \alpha}$ by raising indices. We can now identify $i, j \ldots$ indices with $\alpha, \beta, \ldots$ indices, since they transform in the same way under the $\operatorname{Spin}(5)$ identification. The Grassmann odd parameter $\rho$ will be the "infinitesimal parameter" of the BRST transformation. We note that, the twist breaks the Majorana-Weyl condition on the supersymmetry parameters $\varepsilon_{i}^{\alpha}$ and $\bar{\varepsilon}^{\dot{\alpha} i}$, as indeed it also breaks the Majorana condition in four dimensions in [2]. Moreover, just as we dropped the Majorana-Weyl constraint on the supersymmetry parameter, we also drop it on the spinors of the multiplet, and we regard the scalars $\Phi^{i j}$ and $\bar{\Phi}^{i j}$ as independent fields.

Upon substituting the choice of supersymmetry parameters 2.14), the transformations (2.13) become:

$$
\begin{array}{cc}
\delta B_{\mu \nu}=\rho\left(\gamma_{\mu \nu}\right)_{\alpha}^{\dot{\beta}} \bar{\kappa}_{\dot{\beta}}^{\alpha} & \\
\delta \kappa_{\alpha \beta}=\rho F_{\mu \nu \rho}^{(+)}\left(\gamma^{\mu \nu \rho}\right)_{\alpha \beta} & \delta \bar{\kappa}_{\dot{\alpha}}^{\gamma}=\rho\left(\gamma^{\mu} \partial_{\mu}\right)_{\dot{\alpha} \beta} \bar{\Phi}^{\gamma \beta} \\
\delta \Phi_{i j}=\rho \kappa_{i j}-\frac{1}{4} \Omega_{i j} \rho \kappa_{k}^{k} & \delta \bar{\Phi}_{i j}=0 .
\end{array}
$$

We can expand the spinors $\kappa_{\alpha i} \equiv \kappa_{\alpha \beta}$ and $\bar{\kappa}_{\dot{\alpha}}{ }^{i} \equiv \kappa_{\dot{\alpha}}{ }^{\beta}$ in terms of the complete set of chiral $\gamma$-matrices as follows

$$
\begin{aligned}
& \kappa_{\alpha \beta}=\left(\gamma^{\mu \nu \rho}\right)_{\alpha \beta} \chi_{\mu \nu \rho}^{-1(+)}+\left(\gamma^{\mu}\right)_{\alpha \beta} \eta_{\mu}^{-1} \\
& \kappa_{\dot{\alpha}}^{\beta}=\left(\gamma^{\mu \nu}\right)_{\dot{\alpha}}^{\beta} \Psi_{\mu \nu}^{1}+\delta_{\dot{\alpha}}^{\beta} X^{-1}
\end{aligned}
$$

which makes precise the correspondence between the fermions of the TQFT and those of the supersymmetric theory. The matrices $\left(\gamma^{\mu}\right)_{\alpha \beta}$ are antisymmetric in $\alpha \beta$ while $\left(\gamma^{\mu \nu \rho}\right)_{\alpha \beta}$ are symmetric in $\alpha \beta$ and also antiself-dual. As a result, $\chi_{\mu \nu \rho}^{-1(+)}$ is automatically a self-dual three-form. On the other hand the scalars in $\Phi^{i j} \sim \Phi_{\alpha \beta}$ can be written as

$$
\bar{\Phi}_{\alpha \beta}=\left(\gamma^{\mu}\right)_{\alpha \beta} \Phi_{\mu}^{2} \quad \Phi^{\alpha \beta}=\left(\gamma^{\mu}\right)^{\alpha \beta} \Phi_{\mu}^{-2}
$$

We must also impose the condition $\Phi^{\alpha \beta} \Omega_{\alpha \beta}=0$ and hence there are only ten independent components in $\Phi_{\mu}^{ \pm 2}$. This condition implies that the TQFT multiplet contains two 
more bosons than the supersymmetric one, as indicated by (2.17). This is compensated by the presence of the two extra fermions $\Phi^{ \pm 3}$. (See next section.)

Let us now combine the symmetry (2.15) and the redefinitions (2.16) and (2.17), so as to find the transformations

$$
\begin{aligned}
\delta B_{\mu \nu}=\rho \Psi_{\mu \nu}^{1} \quad \delta \Phi_{\mu}^{2} & =0 \quad \delta \eta_{\mu}^{-1}=0 \\
\delta \Psi_{\mu \nu}^{1}=\rho \partial_{[\mu} \Phi_{\nu]}^{2} \quad \delta X^{1} & =\rho \partial_{\mu} \Phi^{2 \mu} \quad \delta \Phi_{\mu}^{-2}=\rho \eta_{\mu}^{-1} \\
\delta \chi_{\mu \nu \rho}^{-1(+)} & =\rho F_{\mu \nu \rho}^{(+)}
\end{aligned}
$$

In these equations the indices on the fields $\Phi_{\mu}^{ \pm 2}$ only take the values $\mu=1,2, \ldots 5$ and similarly for $\eta_{\mu}$, in order to take into account (2.17).

The comparison to the BRST equations (2.8) also requires $\Phi^{ \pm 3}$ to be set to 0 and the Lagrange multiplier field $H_{\mu \nu \rho}$ to be replaced by the self-duality gauge function for $B_{2}$ and $\eta^{ \pm 2}$ by $\partial_{\mu} \Phi^{\mu \pm 2}$. The latter conditions appear as equations of motion in the $Q$-invariant action that we will consider in the next section (see eqs. (2.21) and (2.24)).

In addition to the above, we must introduce an analogous supersymmetry multiplet and carry out a corresponding twist to reproduce the BRST transformations of the another two-form ${ }^{c} B_{2}$, with similar steps as we did for $B_{2}$ and its ghosts.

\subsection{The Q-invariant and gauge-invariant action.}

The property $Q^{2}=0$ modulo gauge transformations of the two-form $B_{2}$ leads us to consider the following $Q$-invariant and gauge-invariant Lagrangian (the gauge parameter $\alpha$ is a real number, which gives the self-duality condition (2.1) in the limit $\alpha \rightarrow 0$ ):

$$
\begin{gathered}
I_{Q}=\int\left\{Q, \chi^{\mu \nu \rho}\left(\partial_{\mu} B_{\nu \rho}+\epsilon_{\mu \nu \rho \alpha \beta \gamma} \partial_{\alpha}{ }^{c} B_{\beta \gamma}+\alpha H_{\mu \nu \rho}\right)\right. \\
\left.\Phi_{\mu}^{-2} \partial_{\nu} \Psi_{[\mu \nu]}^{1}+\Phi^{-3} \partial_{\mu} \Phi_{\mu}^{2}+X^{1}\left(\eta^{-2}+\partial_{\mu} \Phi_{\mu}^{-2}\right)-c c\right\} .
\end{gathered}
$$

In this expression and the foregoing, the notation $c c$ means terms identical to the explicit ones, which are obtained by replacing all fields with their counterparts with the index ${ }^{c}$. (One must remember that $H_{\mu \nu \rho}$ and ${ }^{c} H_{\mu \nu \rho}$, as well as $\chi_{\mu \nu \rho}$ and ${ }^{c} \chi_{\mu \nu \rho}$, are respectively self-dual and antiself-dual). The action (2.19) indicates our choices for the gauge functions of ghosts and antighosts

$$
\begin{aligned}
& \partial_{\nu} \Psi_{[\mu \nu]}^{1} \\
& \partial_{\mu} \Phi_{\mu}^{2} \\
& \partial_{\mu} \Phi_{\mu}^{-2} .
\end{aligned}
$$


Using the definition of $Q$ and eliminating the fields $H_{\mu \nu \rho}$ and $\eta^{ \pm 2}$ by their algebraic equations of motion

$$
H_{\mu \nu \rho}=\alpha^{-1}\left(\partial_{\mu} B_{\nu \rho}+\epsilon_{\mu \nu \rho \alpha \beta \gamma} \partial_{\alpha}{ }^{c} B_{\beta \gamma}\right)^{(+)} \quad \eta^{ \pm 2}=\partial_{\mu} \Phi_{\mu}^{ \pm 2}
$$

and since:

$$
I_{c l}+\int\left(\left|\partial_{\mu} B_{\nu \rho}+\epsilon_{\mu \nu \rho \alpha \beta \gamma} \partial_{\alpha}^{c} B_{\beta \gamma}\right|^{2}-c c\right)=\int\left(\left|\partial_{\mu} B_{\nu \rho}\right|^{2}-\left|\partial_{\mu}{ }^{c} B_{\nu \rho}\right|^{2}\right)
$$

we get

$$
\begin{gathered}
I_{c l}+I_{Q} \sim \int \alpha^{-1}\left|\partial_{\mu} B_{\nu \rho}\right|^{2}+\chi^{\mu \nu \rho} \partial_{[\mu} \Psi_{\nu \rho]}^{1}+\eta_{\mu}^{-1} \partial_{\nu} \Psi_{[\mu \nu]}^{1}+X^{1} \partial_{\mu} \eta_{\mu}^{-1}+\Phi^{-3} \partial_{\mu} \partial^{\mu} \Phi^{3} \\
+\Phi_{\mu}^{-2} \partial_{\nu} \partial_{[\mu} \Phi_{\nu]}^{2}+\partial_{\nu} \Phi_{\nu}^{-2} \partial_{\mu} \Phi_{\mu}^{2}-c c .
\end{gathered}
$$

The BRST invariance of the action, after elimination of auxiliary fields, is

$$
\begin{array}{r}
Q B_{\mu \nu}=\Psi_{\mu \nu}^{1} \quad Q \Psi_{\mu \nu}^{1}=\partial_{[\mu} \Phi_{\nu]}^{2} \quad Q \Phi_{\mu}^{2}=\partial_{\mu} \Phi^{3} \quad Q \Phi^{3}=0 \\
Q \chi_{\mu \nu \rho}^{-1}=\alpha^{-1}\left(F_{3}+{ }^{* c} F_{3}\right)_{\mu \nu \rho}^{(+)} \quad Q \Phi_{\mu}^{-2}=\eta_{\mu}^{-1} \quad Q \eta_{\mu}^{-1}=0 \\
Q X^{1}=\partial_{\mu} \Phi_{\mu}^{2} \quad Q \Phi^{-3}=\partial_{\mu} \Phi_{\mu}^{-2} .
\end{array}
$$

One has of course all mirror equations obtained by putting the index ${ }^{c}$ on all fields, and $Q^{c} \chi_{\mu \nu \rho}^{-1}=\alpha^{-1}\left(F_{3}+{ }^{* c} F_{3}\right) \stackrel{(-)}{\mu \nu \rho}$.

The two last terms of the action $(2.23)$ can be combined into $\Phi_{\nu}^{-2} \partial^{\mu} \partial_{\mu} \Phi_{\nu}^{2}$. Thus, one has finally:

$$
\begin{aligned}
I_{c l}+I_{Q} \sim I_{\text {top }}=\int & \alpha^{-1}\left|\partial_{\mu} B_{\nu \rho}\right|^{2}-\chi^{\mu \nu \rho} \partial_{[\mu} \Psi_{\nu \rho]}^{1}+\eta_{\mu}^{-1} \partial_{\nu} \Psi_{[\mu \nu]}^{1}-X^{1} \partial_{\mu} \eta_{\mu}^{-1} \\
& -\Phi^{-3} \partial_{\mu} \partial^{\mu} \Phi^{3}+\Phi_{\nu}^{-2} \partial^{\mu} \partial_{\mu} \Phi_{\nu}^{2}-c c .
\end{aligned}
$$

This $S O(5,1)$-invariant $Q$-invariant action, which we have determined by using the self-duality gauge function in(2.1) for the two-form and Feynman-Landau-type gauge functions for the gauge invariances of the topological ghosts, is suitable for defining the TQFT.

To uncover the relation to a supersymmetric action, one must cancel the fermionic ghosts $\Phi^{ \pm 3}$ against, for instance, the components $\Phi_{6}^{ \pm 2}$ of the vector ghosts $\Phi_{\mu}^{ \pm 2}$, as already indicated in section 2.2 at the level of transformation laws. This gives the action

$$
\int \alpha^{-1}\left|\partial_{\mu} B_{\nu \rho}\right|^{2}-\chi^{\mu \nu \rho} \partial_{[\mu} \Psi_{\nu \rho]}^{1}+\eta_{\mu}^{-1} \partial_{\nu} \Psi_{[\mu \nu]}^{1}-X^{1} \partial_{\mu} \eta_{\mu}^{-1}+\sum_{a=1}^{5} \Phi_{a}^{-2} \partial^{\mu} \partial_{\mu} \Phi_{a}^{2}-c c
$$


The compensation between $\Phi^{ \pm 3}$ and $\Phi_{6}^{ \pm 2}$, which we can call "topological packages" as in [7], can in principle be done in a Parisi-Sourlas-type way [9]. The $S O(1,5)$ symmetry now seems broken down to $S O(5) \sim U S P(4)$; as shown in section 2.2 , the restoration of $S O(1,5)$ invariance in the context of $(2,0) D=6$ supersymmetry can be achieved by reinterpreting the ten fields $\Phi_{a}^{ \pm 2}, 1 \leq a \leq 5$, as the ten $S O(1,5)$ scalar components of a complex traceless antisymmetric $U S P(4)$ tensors $\Phi_{i j}$ : then, by doing all other changes of variables (2.16) and (2.17), the action (2.26) reads as a sum of two free six-dimensional actions depending on a self-dual and an antiself-dual (complex) two-form.

Our conclusion is that the free TQFT action (2.23) can be twisted into a free $S O(5,1)$ invariant action depending on a pair of $D=6$ Poincaré two-form multiplets plus a decoupled action depending on two compensating "topological packages". Overall, this mechanism is reminescent of that found in [7], where all superstring actions are connected, by various twists, to a purely topological unifying superstring action with a large enough value of supersymmetry on the worldsheet.

\subsection{Completion of the gauge-fixing.}

We now turn to the gauge-fixing of the remaining ordinary gauge invariances of the action (2.25). The result must be a completely gauge-fixed action, invariant under a completely nilpotent topological BRST symmetry. The ordinary ghost field spectrum of a two-form is

$$
B_{\mu \nu} d x^{\mu} \wedge d x^{\nu}+V_{\mu}^{1} d x^{\mu}+V_{\mu}^{-1} d x^{\mu}+m^{2}+m^{-2}+n .
$$

It indicates that an "on-shell" two-form in six dimensions, with kinetic energy $|\partial B|^{2}$, counts for $C_{4}^{2}=6$ degrees of freedom. $(6=15-2 \cdot 6+3 \cdot 1$. $)$ Once the fields $(2.27)$ are introduced, one combines the BRST operator $Q$ of section 2.3 with the BRST operator for the ordinary gauge symmetries of two-forms. The latter involves the fields in (2.27). This gives a combined nilpotent topological BRST operator $\underline{\mathbf{s}}$, defined as:

$$
\begin{array}{ll}
\underline{\mathbf{s}} B_{\mu \nu}=\Psi_{\mu \nu}+\partial_{[\mu} V_{\nu]} & \underline{\mathbf{s}} V_{\mu}=-\Phi_{\mu}+\partial_{\mu} m \\
\underline{\mathbf{s}} \Psi_{\mu \nu}=\partial_{[\mu} \Phi_{\nu]} & \underline{\mathbf{s}} \Phi_{\mu}=\partial_{\mu} \Phi \\
\underline{\mathbf{s}} \Phi=0 & \underline{\mathbf{s}} m=\Phi .
\end{array}
$$

The possibility of having a geometrical interpretation for the two-form and its ghosts, briefly mentionned in the introduction, relies on the following unifying equation:

$$
(d+\underline{\mathbf{s}})\left(B_{\mu \nu} d x^{\mu} \wedge d x^{\nu}+V_{\mu}^{1} d x^{\mu}+m^{2}\right)=G_{3}+\Psi_{\mu \nu}^{1} d x^{\mu} \wedge d x^{\nu}+\Phi_{\mu}^{2} d x^{\mu}+\Phi^{3},
$$




$$
(d+\underline{\mathbf{s}})\left(G_{3}+\Psi_{\mu \nu}^{1} d x^{\mu} \wedge d x^{\nu}+\Phi_{\mu}^{2} d x^{\mu}+\Phi^{3}\right)=0 .
$$

For the antighosts, one has:

$$
\begin{aligned}
& \underline{\mathbf{s}} \chi_{\mu \nu \rho}^{-1}=H_{\mu \nu \rho} \quad \underline{\mathbf{s}} H_{\mu \nu \rho}=0 \quad \underline{\mathbf{s}} \Phi_{\mu}^{-2}=\eta_{\mu}^{-1} \quad \underline{\mathbf{s}} \eta_{\mu}^{-1}=0 \\
& \underline{\mathbf{s}} \Phi^{-3}=\eta^{-2} \quad \underline{\mathbf{s}} \eta^{-2}=0 \quad \underline{\mathbf{s}} X^{1}=\eta^{2} \quad \underline{\mathbf{s}} \eta^{2}=0 \\
& \underline{\mathbf{s}} V_{\mu}^{-1}=b_{\mu}^{-1} \quad \underline{\mathbf{s}} b_{\mu}=0 \quad \underline{\mathbf{s}} m^{-2}=\beta^{-1} \quad \underline{\mathbf{s}} \beta^{-1}=0 \quad \underline{\mathbf{s}} n=\beta^{1} \quad \underline{\mathbf{s}} \beta^{1}=0 \text {. }
\end{aligned}
$$

The gauge-fixing of the ordinary gauge invariance of the two-form gauge field $B_{\mu \nu}$ and of its ghosts is realized by the following action, which must be added to the action (2.25):

$$
I_{g f}=\int \underline{\mathbf{s}}\left(V_{\mu}^{-1}\left(\partial_{\nu} B_{[\mu \nu]}+\partial_{\mu} n+\frac{1}{2} b_{\mu}\right)+m^{-2} \partial_{\mu} V_{\mu}^{1}-c c\right) .
$$

After expansion and elimination of fields with algebraic equations of motion, one gets:

$$
\begin{aligned}
I_{g f} \sim \int\left|\partial_{\nu} B_{[\mu \nu]}\right|^{2}+\left|\partial_{\mu} n^{0}\right|^{2}+m^{-2} \partial_{\mu} \partial^{\mu} m^{2} \\
\left.\quad-V_{\mu}^{-1} \partial_{[\mu} \partial_{\nu]} V_{\nu}^{1}+\beta^{-1} \partial_{\mu} V_{\mu}^{1}+\beta^{1} \partial_{\mu} V_{\mu}^{-1}-V_{\mu}^{-1} \partial_{\nu} \Psi_{[\mu \nu]}^{1}\right)-c c .
\end{aligned}
$$

By construction, the complete and fully gauge-fixed action $I_{t o p}+I_{g f}$ is invariant under the action of $\underline{\mathbf{s}}$. The last term in (2.34) can be absorbed into a redefinition $\eta_{\mu}^{-1} \rightarrow \eta_{\mu}^{-1}+V_{\mu}^{-1}$ in (2.25), and then $\beta^{1} \rightarrow \beta^{1}+X^{1}$.

\section{Hamiltonian presentation}

To establish a link with the earliest introduction of the Yang-Mills TQFT by Witten [1], we observe that part of the Hamiltonian can be written as $H=\frac{1}{2}\{Q, \bar{Q}\}$, with

$$
\begin{gathered}
Q=\exp -i V\left(\int d^{5} x \Psi_{i j}^{1}(x) \frac{\delta}{\delta B_{i j}(x)}-c c\right) \exp i V-c c \\
\bar{Q}=\exp i V\left(\int d^{5} x \Psi_{i j}^{-1}(x) \frac{\delta}{\delta B_{i j}(x)}-c c\right) \exp -i V \\
V=\int d^{5} x \epsilon^{i j k l m} B_{i j}(x) \partial_{k}{ }^{c} B_{l m}(x) .
\end{gathered}
$$

Here, all indices $i, j$ are five-dimensional. The field $\Psi_{i j}^{-1}(x)$ has 10 components and is replaced by the self-dual three-form $\chi_{\mu \nu \rho}^{-1}(x)$ in six dimensions. Of course, this suggests that there is an interesting five-dimensional action, $\int{ }^{c} B_{2} \wedge d B_{2}$, which should be independently quantized, perhaps by generalizing the method given in [10] for higher-dimensional YangMills Chern-Simons theories [11]. Notice that there is the possibility of adding to ${ }^{c} B_{2} \wedge d B_{2}$ a Yang-Mills- and/or gravitational Chern-Simons- term, since $B_{2}$ can be defined as a gauge field two-form, as noted in the introduction [5]. 


\section{Interacting cases.}

\subsection{Interactions with a Yang-Mills theory through a Chern-Simons coupling.}

Coupling with a Yang-Mills theory can be introduced by adding by adding a ChernSimons term to the Abelian curvature of the two-form, and a Yang-Mills term $\int_{6} \operatorname{Tr}\left|F_{\mu \nu}\right|^{2}$ to the action of the two-forms that we will shortly determine. One defines

$$
G_{3}=d B_{2}+\lambda \operatorname{Tr}\left(A \wedge d A+\frac{2}{3} A \wedge A \wedge A\right),
$$

where $\lambda$ is a constant, possibly adjusted to provide theories with anomaly-compensating mechanisms.

One modifies $Q$ in (2.28) as follows ((2.31) and (2.32) remain the same)

$$
\begin{array}{lll}
\underline{\mathbf{s}} B_{\mu \nu}=\Psi_{\mu \nu}^{1}+\partial_{[\mu} V_{\nu]}^{1}+\lambda \operatorname{Tr}\left(c \partial_{[\mu} A_{\nu]}\right) & \underline{\mathbf{s}} \Psi_{\mu \nu}^{1}=\partial_{[\mu} \Phi_{\nu]}^{2} \quad \underline{\mathbf{s}} \Phi_{\mu}^{2}=\partial_{\mu} \Phi^{3} & \underline{\mathbf{s}} \Phi^{3}=0 \\
\underline{\mathbf{s}} V_{\mu}^{1}=-\Phi_{\mu}^{2}+\partial_{\mu} m^{2}-\lambda \operatorname{Tr}\left(c c A_{\mu}\right) & \underline{\mathbf{s}} m^{2}=\Phi^{3}-\frac{\lambda}{3} \operatorname{Tr}(c c c) . \\
\underline{\mathbf{s}} A_{\mu}=\partial_{\mu} c+\left[A_{\mu}, c\right] & \underline{\mathbf{s}} c=-c c .
\end{array}
$$

One still has $\underline{\mathbf{s}}^{2}=0$. One must complete the BRST equations by $\underline{\mathbf{s}} \bar{c}=b, \underline{\mathbf{s}} b=0$ for the sake of gauge-fixing the ordinary Yang-Mills symmetry. The action is as in (2.19), except that in the gauge function (2.1),$F_{3}=d B_{2}$ is replaced by $G_{3}$. (Notice that $\underline{\mathbf{s}} G_{3}=d \Psi$.) Generalizing what we did in section 2, we get the following $Q$-invariant action

$$
\begin{aligned}
I_{Q} \sim \int & \operatorname{Tr}\left|F_{\mu \nu}\right|^{2}+\frac{1}{\alpha} \mid \partial_{[\mu} B_{\nu \rho]}+\lambda \operatorname{Tr}\left(A \left[\mu \partial_{\nu} A_{\rho]}+\left.\frac{2}{3} A\left[{ }_{\mu} A_{\nu} A_{\rho]}\right)\right|^{2}\right.\right. \\
& -\chi^{\mu \nu \rho} \partial_{[\mu} \Psi_{\nu \rho]}^{1}+\eta_{\mu}^{-1} \partial_{\nu} \Psi_{[\mu \nu]}^{1}-X^{1} \partial_{\mu} \eta_{\mu}^{-1} \\
& +\Phi_{\mu}^{-2} \partial_{\nu} \partial_{[\mu} \Phi_{\nu]}^{2}+\partial_{\nu} \Phi_{\nu}^{-2} \partial_{\mu} \Phi_{\mu}^{2}-\Phi^{-3} \partial_{\mu} \partial^{\mu} \Phi^{3}-c c .
\end{aligned}
$$

This action generalizes (2.25) and contains interactions between $A_{\mu}$, and $B_{\mu \nu}$. As for the link to Poincaré supersymmetry, which holds true in the free case $\lambda=0$, we do not know whether it is compatible with the interacting terms, for the transformation laws and for the action.

\subsection{Coupling to a Yang-Mills TQFT.}

In six dimensions, there is a Higgs Yang-Mills TQFT 四. Rougly speaking, this theory is a dimensional reduction of the eight-dimensional pure Yang-Mills TQFT with octonionic self-duality equations as gauge functions, for which the topological BRST symmetry is also related to Poincaré supersymmetry. Its action can be substituted to $\operatorname{Tr}\left|F_{\mu \nu}\right|^{2}$ in (4.3). One must modify (4.2) to take into account the transformation law of the Yang-Mills field as given in [4]. This gives new ghost interactions which modifies (4.3). 


\section{Higher Dimensions than six.}

Here we consider the TQFT for a self-dual three-form in eight dimensions. Formula (2.7) becomes:

$$
\begin{aligned}
& F_{\mu \nu \rho \sigma} d x^{\mu} \wedge d x^{\nu} \wedge d x^{\rho} \wedge d x^{\sigma}+\Psi_{\mu \nu \rho}^{1} d x^{\mu} \wedge d x^{\nu} \wedge d x^{\rho}+\chi_{\mu \nu \rho \sigma}^{-1} d x^{\mu} \wedge d x^{\nu} \wedge d x^{\rho} d x^{\sigma} \\
& +\Phi_{\mu \nu}^{2} d x^{\mu} \wedge d x^{\nu}+\Phi_{\mu \nu}^{-2} d x^{\mu} \wedge d x^{\nu}+\Phi_{\mu}^{3} d x^{\mu}+\Phi_{\mu}^{1} d x^{\mu}+\Phi_{\mu}^{-3} d x^{\mu}+\Phi^{4}+\Phi^{2}+\Phi^{-2}+\Phi^{-4}
\end{aligned}
$$

$\chi_{\mu \nu \rho \sigma}^{-1}$ is a self-dual four-form antighost adapted for the eight-dimensional self-duality condition of the curvature of $B_{3}$. Notice that there is no need to double the degrees of freedom as in six dimensions, since a single three-form determines the invariant $\int_{8} d B_{3} \wedge d B_{3}$. By generalizing what we did in six dimensions, we obtain fermionic Lagrange multipliers fields that are a two-form $\eta_{\mu \nu}^{-1}$ and three zero-forms $\eta^{ \pm 3}$ and $\eta^{3}$. Eventually, the TQFT multiplet (2.9) becomes

$$
\left(B_{\mu \nu \rho}, \Phi_{\mu \nu}^{ \pm 2}, \Phi^{ \pm 4}, \Phi^{ \pm 2}, \Psi_{\mu \nu \rho}^{1}, \chi_{\mu \nu \rho \sigma}^{-1}, \eta_{\mu \nu}^{-1}, \Phi_{\mu}^{ \pm 3}, \Phi_{\mu}^{1}, \eta^{ \pm 3}, \eta^{1}\right)
$$

The number of degrees of freedom of the TQFT, in addition to that of the 3 -form $B_{\mu \nu \rho}$, is $2 \cdot 28+4=60$ for the bosons and $56+35+28+3 \cdot 8+3=146$ for the fermions. This set of fields can be twisted into the $\mathrm{D}=8$ supermultiplet of a three-form made of two two-forms, two one-forms, two zero-forms, and one gravitino and three spinors

$$
\left(B_{\mu \nu \rho}, 2 A_{\mu \nu}, 2 A_{\mu}, 2 \Phi, 1 \Psi_{\mu}^{\alpha}, 3 \kappa^{\alpha}\right)
$$

This $N=1$ multiplet has on-shell states that are given by tensor product of the on-shell states of the $N=1$ Yang-Mills multiplet with the $4+\overline{4}$ of the little group $S O(6)$. Let us indeed check how the counting of degrees of freedom of the supersymmetric multiplet (5.3) matches that of the TQFT in (5.2). When counting the number of degrees of freedom we must as usual subtract the ones which are pure gauges. The number of boson degrees of freedom is thus $7 \cdot 6=42$ for both two-forms, $7 \cdot 2=14$ for the two one-forms and 2 for both zero-forms, which makes 58 boson degrees of freedom to be added to those of the three-form. For the gravitino and three spinors, the spinor index $\alpha$ runs over 16 values. Thus we have $6 \cdot 16=96$ degrees of freedom for the gravitino and $3 \cdot 16=48$ ones for the three spinors, that is $144=96+48$ fermionic degrees of freedom. If, as in the six-dimensional case, we eliminate a pair of bosons against a pair of fermions in (5.2), for instance $\Phi^{ \pm 4}$ against $\eta^{ \pm 3}$, we get an exact matching between the fields in the TQFT system 
(5.2) and the field of the supersymmetric system (5.3), with 58 bosons and 144 fermions. This indicates the possibility of a twist between the two systems. The striking difference between the situations in six and eight dimensions is the apparition of the gravitino in eight dimensions, which gives a supersymmetric theory depending on a spin- $3 / 2$ field with no gravity coupling. Again, interactions with Yang-Mills field can be introduced by adding Chern classes in the self-duality equations, while the Yang-Mills dynamics can be defined from an eight-dimensional TQFT as in [4].

Preliminary computations also suggest that the TQFT system in ten dimensions for a self-dual four-form might be twistable into the supersymmetric multiplet of type IIB supergravity.

\section{Conclusion}

We have shown that the notion of a twist between TQFTs and supersymmetric theories can be generalized from that known to exist in two and four dimensions provided one eliminates topological pairs. In particular, we have considered a topological theory of twoforms in six dimensions and established the relation of its BRST symmetry to the twisted transformations of the tensor supermultiplet of $(2,0)$ Poincaré supersymmetry. Although there are a number of features of the twisting procedure that remain mysterious, it is apparent that there is a general relationship between supersymmetry transformations and the BRST transformations associated with topological theories. Central to this relationship is the fact that TQFTs involve self-duality conditions as their gauge-fixing conditions and that self-duality conditions arise naturally in supersymmetric theories as a consequence of the intimate relationship between self-duality, supersymmetry and spinor chirality. It is interesting to note that the six and four dimensional supersymmetric theories both contain spinor fields which satisfy a symplectic Majorana condition.

We have also presented interactive theories that could be interesting to explore. In eight dimensions, there are indications that these features repeat themselves for the theory of three-forms, leading, after a twist, to a theory with a gravitino, but no graviton.

An intriguing open question is to examine if another expression of the six-dimensionalTQFT can be untwisted in the action recently proposed in [12 for self-dual two-forms.

\section{Acknowledgements}

We are grateful to E. Cremmer for most useful and interesting discussions related to this work. 


\section{References}

[1] E. Witten, Topological Quantum Field Theory, Commun. Math.Phys. 117 (1988)353.

[2] E. Witten, Supersymmetric Yang-Mills theory on a four-manifold, J. Math. Phys. 35 (1994) 5101.

[3] L. Baulieu, I. M. Singer, Topological Yang-Mills Symmetry, Nucl. Phys. Proc. Suppl. 15B (1988) 12;

L. Baulieu, On the Symmetries of Topological Quantum Field Theories, hep-th/ 9504015, Int. J. Mod. Phys. A10 (1995) 4483;

R. Dijkgraaf, G. Moore, Balanced Topological Field Theories, hep-th/9608169, Commun. Math. Phys. 185 (1997) 411.

[4] L. Baulieu, H. Kanno, I. Singer, Special Quantum Field Theories in Eight and Other Dimensions, hep-th/9704167, Talk given at APCTP Winter School on Dualities in String Theory, (Sokcho, Korea), February 24-28, 1997;

L. Baulieu, H. Kanno, I. Singer, Cohomological Yang-Mills Theory in Eight Dimensions, hep-th/9705127, to appear in Commun. Math.Phys.

[5] J. Cheeger and J. Simons, Differential Characters and Geometric Invariants, Stony Brook Preprint, (1973), unpublished.

[6] L. Baulieu, Field Antifield Duality, p-Form Gauge Fields and Topological Quantum Field Theories, hep-th/9512026, Nucl.Phys. B478 (1996) 431.

[7] L. Baulieu, M. B. Green, E. Rabinovici A Unifying Topological Action for Heterotic and Type II Superstring Theories, hep-th/9606080, Phys.Lett. B386 (1996) 91;

Superstrings from Theories with $N>1$ World Sheet Supersymmetry, hep-th/9611136, Nucl. Phys. B498 (1997).

[8] A. Salam and E. Sezgin, Supergravities in diverse dimensions, vol. 1, p.119;

P. Howe, G. Sierra and P. Townsend, Nucl Phys B221 (1983) 331

[9] G. Parisi and N. Sourlas, Random Magnetic Fields, Supersymmetry and Negative Dimensions, Phys. Rev.Lett. 43 (1979) 744;

Nucl. Phys. B206 (1982) 321.

[10] A. Losev, G. Moore, N. Nekrasov, S. Shatashvili, Four-Dimensional Avatars of TwoDimensional RCFT, hep-th/9509151, Nucl. Phys. Proc. Suppl. 46 (1996) 130;

L. Baulieu, A. Losev, N. Nekrasov Chern-Simons and Twisted Supersymmetry in Higher Dimensions, hep-th/9707174, to appear in Nucl. Phys. B.

[11] L. Baulieu and E. Rabinovici, in preparation.

[12] G. Dall'Agata, K. Lechner and M. Tonin, Covariant actions for $N=1, D=6$ Supergravity theories with chiral bosons, hep-th/9710127, Nucl. Phys. B512 (1998) 179-198. 